Revista Destaques Acadêmicos, Lajeado, v. 8, n. 3, 2016. ISSN 2176-3070 DOI: http://dx.doi.org/10.22410/issn.2176-3070.v8i3a2016.1057 www.univates.br/revistas

\title{
DETERMINAÇÃO DE TEOR E UNIFORMIDADE DE DOSES DE CÁPSULAS DE BESILATO DE ANLODIPINO MANIPULADAS EM FARMÁCIAS DE VENÂNCIO AIRES-RS
}

\author{
Daiana Kipper Manganelli ${ }^{1}$, Luisa Scheer Ely ${ }^{2}$, Renata Vidor Contri ${ }^{3}$
}

\begin{abstract}
Resumo: A hipertensão arterial é um problema de saúde pública que resulta no aumento da probabilidade de doenças cardiovasculares e cerebrais. O besilato de anlodipino é um dos tratamentos medicamentosos mais utilizados, sendo frequentemente administrado a partir de formulações manipuladas com baixa dosagem de princípio ativo por unidade farmacotécnica. O presente trabalho teve por objetivo avaliar o teor e a uniformidade de doses de cápsulas de besilato de anlodipino manipuladas por farmácias da cidade de Venâncio Aires- RS. As amostras de 3 farmácias testadas apresentaram valores adequados, de acordo com as especificações farmacopeias, comprovando que cápsulas manipuladas podem ser confiáveis com relação à quantidade de fármaco declarado no rótulo, desde que sejam seguidas as boas práticas de manipulação.
\end{abstract}

Palavras-chave: Hipertensão. Besilato de anlodipino. Teor. Uniformidade de doses. Boas práticas de manipulação.

\section{INTRODUÇÃO}

A hipertensão arterial, na maioria das vezes, não causa nenhum sintoma, sendo considera como uma doença multicausal e multifatorial. Alguns fatores como idade, raça, sexo, alimentação inadequada, sedentarismo e genética podem ocasionar o aumento da pressão arterial. A hipertensão está associada a outras doenças que trazem repercussões negativas para a qualidade de vida do indivíduo, como doenças cardiovasculares e cerebrais, dentre estas o acidente vascular encefálico, uma das principais causas de morte. (SARAIVA et al.,2007) (ACHUTTI; ACHUTTI, 1997). Segundo dados do Ministério da Saúde de 2011, estima-se que a hipertensão arterial atinge cerca de $25 \%$ da população brasileira, causando um grande problema de saúde pública. Segundo a

1 Acadêmica do Curso de Farmácia do Centro Universitário UNIVATES.

2 Docente Doutora do Curso de Farmácia do Centro Universitário UNIVATES.

3 Docente Doutora do Curso de Farmácia do Centro Universitário UNIVATES. 
Sociedade Brasileira de Hipertensão (SBH), a pressão ideal para minimização do risco de problemas cardiovasculares situa-se abaixo de $120 / 80 \mathrm{mmHg}$ com ressalvas para diabéticos $(130 / 85 \mathrm{mmHg})$ e renais crônicos $(120 / 75 \mathrm{mmHg})$.

Quando medidas alternativas, como cuidado com a alimentação e exercícios físicos, não são eficazes para a diminuição da pressão, é necessário que os pacientes procurem o médico para uma nova avaliação e façam, caso necessário, o uso de medicamentos conhecidos como anti-hipertensivos. A escolha do medicamento tem como objetivo principal reduzir a mortalidade e morbidade em pacientes com hipertensão. Para que isso ocorra, sempre deverá ser assegurado que o medicamento seja eficaz e seguro (SOCIEDADE BRASILEIRA DE CARDIOLOGIA, 2015).

Um dos medicamentos mais utilizados para o controle da hipertensão é o besilato de anlodipino, um bloqueador de canal de cálcio ou também conhecido como antagonista do canal de cálcio. Este provoca a diminuição da entrada de cálcio para dentro das células mióticas dos músculos lisos e cardíacos, provocando a despolarização e o relaxamento no músculo liso vascular, permitindo que o sangue flua mais facilmente, diminuindo assim a frequência cardíaca e excitação. $\mathrm{O}$ besilato de anlodipino pode ser classificado como um anti-hipertensivo de terceira geração por ser uma dihidropiridina, tendo maior efeito e maior seletividade em comparação com outros antihipertensivos. O besilato de anlodipino é o mais seguro desta classe, podendo assim fazer associação deste com outros anti-hipertensivos (GOODMAN; GILMAN, 2010). É um medicamento de uso oral, se encontrando disponível no mercado como comprimidos sulcados com apresentação comercial de 2,5, 5 e $10 \mathrm{mg}$. Outra opção é a obtenção de cápsulas magistrais produzidas em farmácias de manipulação (SILVA; CHOZE; ANDRADE; SILVA,2014).

A procura por medicamentos preparados nas farmácias magistrais se deve a sua ampla diversificação de produtos para as necessidades específicas de cada paciente, além de serem produzidos em pequena escala, com tecnologia simples e com menos testes de qualidade realizados, o que pode tornar o produto mais acessível para a população. Outras vantagens incluem produção de associações, dosagens e formas farmacêuticas não disponíveis no mercado (LACERDA, 2014; FERNANDES, 2015)

Os produtos manipulados devem seguir as boas práticas de manipulação (BRASIL, RDC 67 / 2007). Sabe-se que a realização do controle de qualidade das formulações magistrais é importante para comprovação da segurança e eficácia destes medicamentos. A dose incorreta do medicamento pode levar à ineficácia terapêutica do produto manipulado, bem como ao aumento dos efeitos adversos e/ou tóxicos. Para assegurar a quantidade de princípio ativo presente na forma farmacêutica, é necessário realizar análise de teor e de uniformidade de doses. (COUTO; TAVARES, 2010). Estas análises são de extrema importância principalmente em casos onde o princípio ativo se encontra em baixa dosagem na forma farmacêutica, como é o caso do besilato de anlodipino. 
Baseado no exposto, o presente trabalho teve por objetivo analisar o teor e a uniformidade de doses de cápsulas contendo besilato de anlodipino manipuladas em distintas farmácias de manipulação do município de Venâncio Aires - RS.

\section{MATERIAIS E MÉTODOS}

\subsection{Materiais}

As cápsulas de besilato de anlodipino $5 \mathrm{mg}$ foram compradas em três farmácias de manipulação na cidade de Venâncio Aires-RS, na quantidade de 60 cápsulas por farmácia, estando estas dentro do prazo de validade. As farmácias foram nomeadas como A, B e C. Como substância padrão, foi utilizado besilato de anlodipino Fagron (Brasil), lote AMB/022/08/12 com validade 01/07/2017. Também foram utilizados metanol e ácido clorídrico de padrão analítico e água purificada.

\subsection{Método analítico para quantificação de besilato de anlodipino}

O método utilizado foi previamente descrito e validado por Malesuiket al. (2005). A absorção de ambas soluções (padrão e amostra) foram avaliadas em 238 nm (Espectrofotômetro Evolution 60S, EUA) conforme descrito como comprimento de onda de máxima absorção pela Farmacopeia Britânica, que descreve a análise de absorção no ultravioleta como teste de identificação do besilato de anlodipino. Foi utilizada uma cubeta de quartzo de $1 \mathrm{~cm}$ e água purificada para zerar o equipamento.

\subsection{Solução padrão de besilado de anlodipino}

Inicialmente preparou-se uma solução padrão de besilato de anlodipino pesando-se $10 \mathrm{mg}$ da substância e transferindo a quantidade pesada para um balão volumétrico de $100 \mathrm{ml}$ acrescentando metanol P.A. Esta solução novamente foi diluída com água purificada até atingir a concentração $20 \mu \mathrm{g} / \mathrm{ml}$ de besilato de anlodipino, que foi utilizada para análise do teor das cápsulas. Outras 4 concentrações $(5,10,15,25 \mu \mathrm{g} / \mathrm{ml})$ foram avaliadas para o estudo da linearidade do método.

Para a correção da concentração do padrão, foi analisada a umidade presente no padrão analítico utilizando uma balança de infravermelho no modo automático (MARTE, São Paulo - SP), além de ser considerado o valor de teor em base anidra descrito no laudo.

\subsection{Extração do besilato de anlodipino a partir de cápsulas manipuladas}

Foi removido o conteúdo das cápsulas e feita a extração com banho de ultrassom (15 min.) (UltrasonicCleaner, Unique, Brasil) utilizando1\% $\mathrm{HCl}$ 0,1 N 
em metanol (V/V), seguido de centrifugação (3000 rpm por $10 \mathrm{~min}$.) (Macro Ev: 04, Brasil) para separação dos sólidos não dissolvidos. A segunda diluição, até dosagem final de $20 \mu \mathrm{g} / \mathrm{ml}$ de besilato de anlodipino, foi realizada transferindo o sobrenadante resultante da centrifugação e diluindo com água purificada. Foi considerado o valor de equivalência de 1,39 para que seja obtida a concentração final desejada do besilato de anlodipino extraído das cápsulas.

\subsection{Teor das cápsulas de besilato de anlodipino}

A avaliação do teor, realizada conforme a Farmacopeia Brasileira (2010) foi realizada com 20 cápsulas onde foi retirado o conteúdo destas e em seguida homogeneizado para se obter um pool. Amostras $(n=3)$ do pool foram extraídas conforme item acima. A absorção observada no espectrofotômetro foi avaliada de forma comparativa à absorção observada pelo padrão, conforme a equação 1 descrita abaixo. Foi considerado aprovado neste teste aquelas amostras que apresentaram teor entre 90 e $110 \%$.

Será utilizada a equação abaixo:

$\%$ de fármaco $=\underline{(\mathrm{Aa} \times \mathrm{Cp} \times 100)}$

$(\mathrm{Ap} \times \mathrm{Ca})$

Sendo que $\mathrm{Aa}=$ absorbância da amostra, $\mathrm{Cp}=$ concentração do padrão corrigido pelo teor descrito no laudo e pela umidade em $\mathrm{ug} / \mathrm{mL}, \mathrm{Aa}=$ Absorção da amostra e $\mathrm{Ca}=$ concentração teórica da amostra em ug $/ \mathrm{mL}$.

\subsection{Uniformidade de doses de cápsulas de besilato de anlodipino}

Para o teste de uniformidade de dose, realizado conforme a Farmacopeia Brasileira (2010) foram separadas inicialmente 30 cápsulas. Destas, foram separadas 10 amostras aleatórias. As amostras foram avaliadas segundo o seu conteúdo individualmente, utilizando o método de espectrofotometria no UV previamente mencionado. Uma a uma, as cápsulas tiveram seu conteúdo removido e analisado. A absorção observada no espectrofotômetro foi avaliada de forma comparativa à absorção observada pelo padrão, conforme a equação 1 descrita acima. Foi considerado aprovado neste teste aquelas amostras que apresentaram valor de aceitação (VA) de até 15 conforme descrito na Farmacopeia Brasileira (2010).

\section{RESULTADOS E DISCUSSÃO}

Uma das formas farmacêuticas mais utilizadas pelas farmácias de manipulação são as cápsulas duras, devido à facilidade de produção e de ajuste às necessidades de cada paciente. Outro importante fator é a boa biodisponibilidade das cápsulas que rapidamente são dissolvidas, liberando o fármaco mais rápido para chegar ao seu local de ação. O processo acontece 
em torno de 10 a 20 minutos ao contrário dos comprimidos que levam mais tempo para se dissolverem (LE HIR, 1995; VILLA-JATO, 1997). Dentre os testes importantes de serem realizados para garantir a qualidade de cápsulas estão o aspecto, peso médio, teor e uniformidade de doses.

Os testes de teor e uniformidade de doses têm por objetivo avaliar se a concentração do fármaco na cápsula está correta, para garantir e assegurar que a dose esteja correta comparada com a dose declarada. $\mathrm{O}$ teor é avaliado a partir da mistura do conteúdo de diversas cápsulas, formando um pool. A uniformidade de doses unitárias tem como objetivo avaliar se a distribuição da substância ativa está uniforme em cada unidade do lote analisado. Estes testes não são normalmente realizados em farmácias de manipulação, sendo estes muitas vezes terceirizados para alguns lotes apenas. Para a realização dos testes é necessário um método de análise quantitativa adequado.

O método utilizado neste trabalho para as análises quantitativas do besilato de anlodipino baseou-se na absorção na faixa do ultravioleta utilizando espectrofotômetro, previamente descrito e validado por Malesuik et al. (2005). O método foi considerado sensível, linear e específico, para analise em comprimidos e em cápsulas (MALESUIK et al., 2005).

O espectrofotômetro ultravioleta (UV) é frequentemente aplicado como método de identificação e quantificação de fármacos, apresentando praticidade, baixo custo, simples interpretação dos dados e relativa sensibilidade e especificidade (GALO; COLOMBO, 2009). Este método se baseia na absorção de energia de acordo com a estrutura da molécula e sua concentração (SILVERSTEIN; BASSLER; MORRILL,1981).

Previamente ao início das análises das cápsulas manipuladas, foi realizado o estudo da linearidade do método, avaliando quatro concentrações diferentes ( $5 \mathrm{mg}, 10 \mathrm{mg}$, 15mg, 20mg e $25 \mathrm{mg}$ ). A absorbância foi medida em duplicata e realizada uma média dos valores. Foram avaliadas as absorções das amostras em $238 \mathrm{~nm}$ conforme descrito para o método analítico. Os valores foram aplicados na equação da reta, verificando-se uma boa correlação entre os dados $\left(R^{2}=0,9981\right)$ (FIGURA 1): 
Figura 1- Curva analítica do método por absorção no ultravioleta para quantificação do besilato de anlodipino

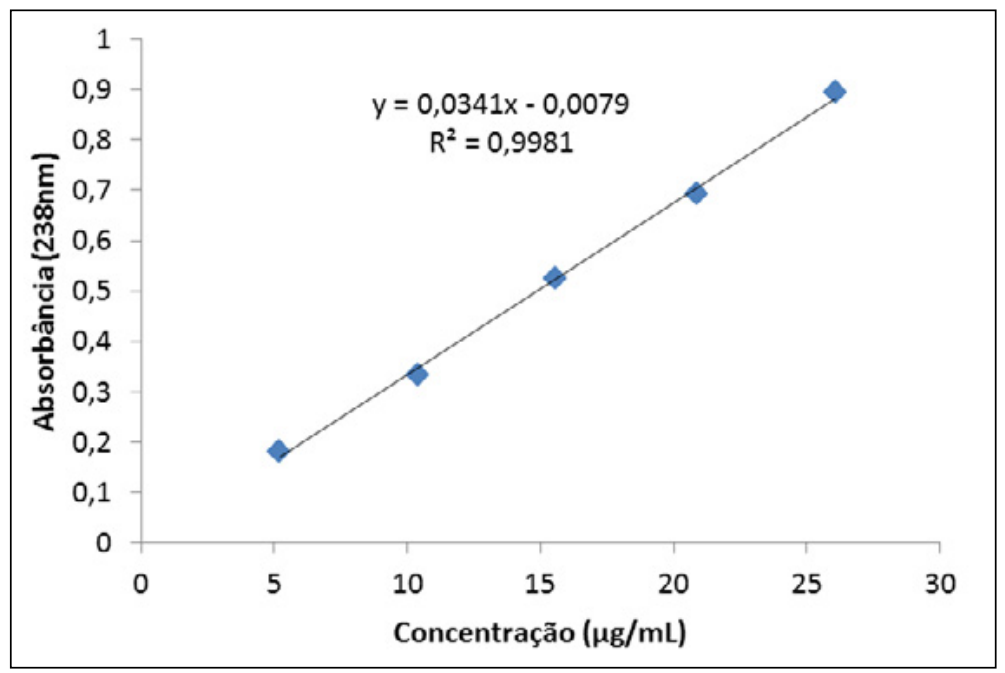

Os valores relacionados à linearidade mostram que os resultados de absorção são correlacionáveis com a concentração do besilato de anlodipino, confirmando que o método previamente validado é adequado para ser aplicado ao doseamento de besilato de anlodipino.

As análises de teor de besilato de anlodipino aponta que as três farmácias analisadas apresentaram valor dentro da faixa de 90 e $110 \%$. A faixa foi determinada de acordo com a monografia da farmacopéia americana (USP 38 , 2015) para os comprimidos de besilato de anlodipino, uma vez que não existem monografias para cápsulas ou comprimidos de besilato de anlodipino na farmacopéia brasileira. Através da Figura 2 pode-se observar a diferenças entre as farmácias analisadas. 
Figura 2 - Análise do teor de cápsulas manipuladas de besilato de anlodipino (média \pm desvio padrão para 3 análises do pool de 20 cápsulas)

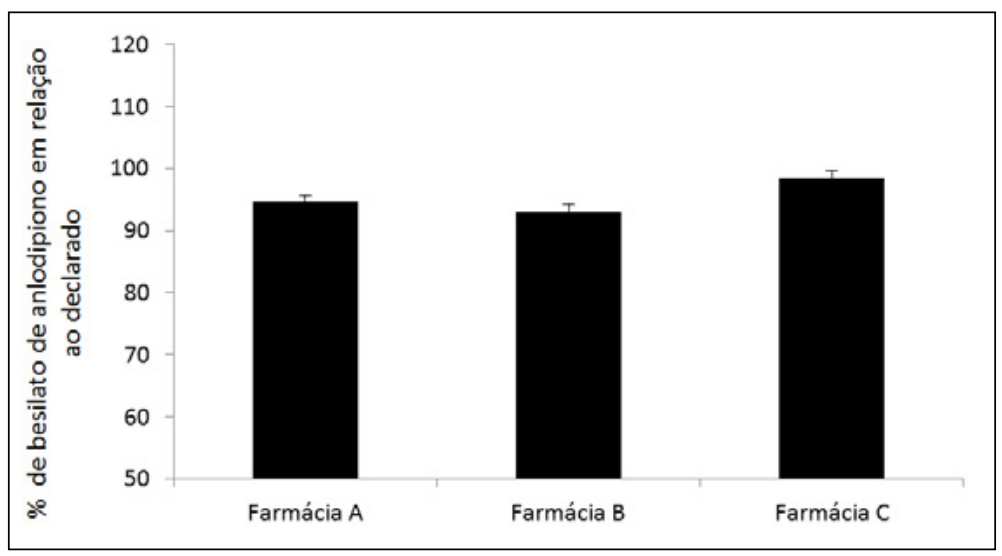

Para garantir a quantidade de substância ativa declarada em cada unidade de dosagem, deve ser realizado o teste de uniformidade de doses além da análise de teor. Este teste apresenta duas formas de avaliação, uniformidade de conteúdo e variação de peso. Na segunda, não há uma quantificação de cada unidade de cápsula ou comprimido, mas sim uma estimativa do doseamento através do peso. O que determina o teste a ser aplicado é a forma farmacêutica, a dose e a proporção do fármaco.

O teste de variação de peso não se aplica para todas as formas farmacêuticas e sim somente para cápsulas duras e comprimidos tanto revestidos ou não revestidos que contenham $25 \mathrm{mg}$ ou mais de princípio ativo e desde que a proporção de ativo na forma farmacêutica seja de pelo menos 25 $\%$. Conforme a Farmacopeia Brasileira (2010), para o presente trabalho o teste que melhor se enquadra para as cápsulas de besilato de anlodipino é o método por uniformidade de conteúdo, pois as cápsulas de besilato de anlodipino que será analisado apresenta uma dosagem de $5 \mathrm{mg}$ (FARMACOPEIA, 2010). Desta forma, foi analisada a concentração do componente ativo individualmente em cada cápsula verificando se, a partir dos valores de doseamento, o valor de aceitação calculado se enquadra dentro dos limites esperados.

Inicialmente, são apresentados os valores médios do doseamento das 10 cápsulas das diferentes farmácias (FIGURA 3), para o teste de uniformidade de doses. Assim como para o teor, as análises apresentaram valores médios próximo a $100 \%$. 
Figura 3 - Análise da Uniformidade de doses (média \pm desvio padrão para 10 análises de cápsulas independentes)

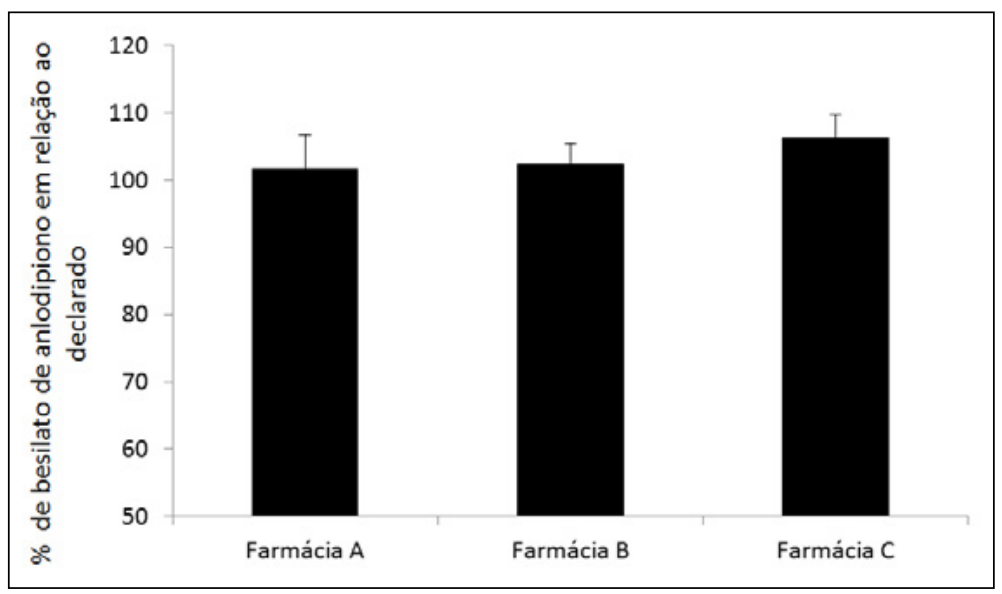

Porém, para verificar se as cápsulas cumprem o teste de uniformidade de doses, é necessário o cálculo do valor de aceitação. O valor máximo permitido para o valor de aceitação para que as cápsulas sejam consideradas aprovadas é de 15, considerando a análise de 10 cápsulas inicialmente. Como podemos verificar na tabela 1, as amostras avaliadas das 3 farmácias ficaram dentro do valor limite de aceitação, sendo consideradas aprovadas. A farmácia $C$ teve o valor de aceitação mais elevado em comparação com as outras farmácias, porém ainda dentro do limite de 15.

Tabela 1- Determinação do valor de aceitação durante análise de uniformidade de doses

\begin{tabular}{lccc}
\hline Farmácia & $\begin{array}{c}\text { Média dos 10 } \\
\text { doseamentos }\end{array}$ & $\begin{array}{c}\text { Desvio padrão dos } 10 \\
\text { doseamentos }\end{array}$ & Valor de aceitação \\
\hline A & 101,7 & 4,92 & 12,02 \\
B & 102,3 & 3,21 & 8,45 \\
C & 106,2 & 3,44 & 13,00 \\
\hline
\end{tabular}

De modo geral, os bloqueadores do canal de cálcio, como é o caso do besilato de anlodipino, são considerados os mais seguros para o controle da hipertensão, podendo ser associados com outros anti-hipertensivos. Porém, efeitos adversos como tonturas, cefaléia, hipotensão e náuseas são muito comuns devido à sua vasodilatação excessiva (GOODMAN; GILMAN, 2010). O besilato de anlodipino é considerado fármaco de terceira geração por apresentar tempo de meia vida maior, ou seja, uma longa duração do medicamento. Outra vantagem deste fármaco de terceira geração é que este apresenta menor 
ocorrência de taquicardia em paciente que fazem uso de outros medicamentos pertencentes aos antagonistas de canais de cálcio (RIBEIRO; MUSCARÁ, 2001).

Conforme Malesuik e colaboradores (2005), as cápsulas manipuladas e comprimidos contendo besilato de anlodipino apresentam resultados dentro das especificações, a exceção de um lote de cápsulas que apresentou falta de uniformidade de doses após 30 unidades testadas. Já de acordo com Silva e colaboradores (2013), que avaliaram cápsulas manipuladas, comprimidos e comprimidos partidos de besilato de anlodipino, as cápsulas manipuladas não apresentaram valores adequados de uniformidade de doses. Segundo o autor, a falta de padronização de técnicas e matérias-primas é um problema que afeta o setor magistral (SILVA; CHOZE; ANDRADE; SILVA,2014).

Diversos outros fármacos já foram analisados quanto ao teor e/ou uniformidade de doses em cápsulas manipuladas apresentando resultados satisfatórios, como o maleato de enalapril (KÜLKAMP et al., 2011), o captopril (MARCATTO et al., 2006), a furosemida (LAMOLHA et al., 2011), o propranolol (RIGOBELLO et al., 2013) e a fomotidina (FAVORETTO et al., 2010), indicando a qualidade das cápsulas manipuladas.

Por serem medicamentos produzidos em pequena escala, com tecnologias mais simples, e submetidos a menos ensaios de qualidade, os medicamentos manipulados costumam se tornar mais acessíveis, ou seja, mais baratos para a população, quando comparados com os medicamentos industrializados. Outras vantagens incluem produção de associações, dosagens e formas farmacêuticas não disponíveis no mercado (LACERDA, 2014, FERNANDES, 2015). Devido à grande procura pelos medicamentos magistrais foi homologada a RDC $33 \mathrm{em}$ 19 de abril de 2000, também conhecida como Boas Práticas de Manipulação em Farmácia (BPMF), com intuito de realizar uma fiscalização no controle do produto visando garantir a qualidade da fórmula magistral. Foi atualizada e se tornou a RDC 67/2007, a qual se encontra em vigor até os dias de hoje (BONFILIO; EMERICK; NETTO JR.; SALGADO, 2011).

Conforme a RDC 67/2007, a farmácia deverá garantir e assegurar a qualidade dos produtos manipulados tanto no seu preparo como na dispensação. Para que isso ocorra de maneira correta é necessário que o profissional farmacêutico esteja presente em todas as etapas para garantir a qualidade do produto; demonstrar uma estrutura adequada do local para o manuseio dos medicamentos, qualificando funcionários através de treinamentos periódicos sem deixar de ter uma quantidade suficiente destes, além disso, sempre mantêlos motivado para que cumpra os padrões de qualidade (BRASIL, RDC n ${ }^{\circ} 67$ ). Os resultados obtidos neste trabalho com cápsulas de besilato de anlodipino, bem como em trabalhos anteriores avaliando outros fármacos mostram a qualidade de cápsulas obtidas em farmácias de manipulação, quanto à quantidade de princípio ativo presente. 


\section{CONCLUSÃO}

Os resultados obtidos nos testes teor e uniformidade de doses de cápsulas manipuladas de besilato e anlodipino obtidas em farmácias de Venâncio AiresRS encontram-se de acordo com as especificações farmacopeias estipuladas para as análises. Desta forma, é mostrado que as cápsulas magistrais podem ser medicamentos confiáveis, com relação a quantidade de fármaco declarado no rótulo, desde que sejam seguidas as boas práticas de manipulação.

\section{REFERÊNCIAS}

ACHUTTI, A.C; ACHUTTI, V.A.R. Aspectos epidemiológicos. In AMODEU, C.; LIMA, E.G., VAZQUEZ, E.C. Hipertensão arterial. São Paulo, 1997.

ANVISA. AGENCIA NACIONAL DE VIGILÂNCIA SANITÁRIA. Farmacopeia Brasileira. 5 ed. Vol. 1 e 2. Brasília: Agência Nacional de Vigilância Sanitária, 2010.

BONFILIO, Rudy; EMERICK, Gulherme L.; NETTO JR., Antônio; SALGADO, Hérida R. N. FARMÁCIA MAGISTRAL: SUA IMPORTÂNCIA E SEU PERFIL DE QUALIDADE. Rev Baiana Saude Publica Miolo.,v. 34,n.3, 2011. Disponível em: <http:/ /files.bvs.br/upload/S/0100-0233/2010/v34n3/a1874.pdf>. Acesso em: 12 nov. 2015.

BRASIL. Resolução - RDC n ${ }^{\circ} 33$, de 19 de abril de 2000(*). Regulamento Técnico sobre Boas Práticas de Manipulação de Medicamentos em farmácias. Disponível em: <http://www.cff.org.br/userfiles/file/resolucao_sanitaria/33_19abril.pdf $>$. Acesso em: 09 nov. 2015.

BRASIL. Resolução de Diretoria Colegiada (RDC) 67, de 08 de outubro de 2007. Dispõe sobre as Boas Práticas de Manipulação de Medicamentos para Uso Humano em Farmácias e seus anexos. Diário Oficial da União, 09 out. 2007. Disponível em: $<$ http:/ / www.brasil.gov.br/saude/2012/04/remedios-manipulados-sao-ate-20-maisbaratos-que-os-industrializados>. Acesso em: 09 set. 2015.

BRASIL.Saúde anuncia dados da hipertensão no País. Disponível em: $<$ http:/ /www. brasil.gov.br/saude/2011/04/saude-anuncia-dados-da-hipertensao-no-pais $>$. Acesso em: 09 set. 2015.

COUTO, A. G.; TAVARES, R. C. Análise do perfil dos resultados de uniformidade de conteúdo de cápsulas de baixa dosagem produzidas em farmácias de manipulação de Santa Catarina. Rev. Ciênc. Farm. Básica Apl., v. 32, n. 1, p. 263-268, 2011.

FAVORETTO, Lívia B. et al. Validação de método espectrofotométrico na região do UV para quantificação de famotidina em cápsulas. Quim. Nova, v. 33, n. 7, p. 1585$1589,2010$. 
FERNANDES, Cristiane K. C. Análise do perfil de utilização de medicamentos manipulados no município de Goiânia/GO. Dissertação (Especialização), 95 f. Faculdades Alves Faria - ALFA, Goiânia, 2015.

GALO, A. L.; COLOMBO, M. F. Quim. Nova., 2009.

GOODMAN; GILMAN. Manual de Farmacologia e Terapêutica. 1. ed. Artmed, 2010.

KÜLKAMP, Irene C. et al. Desenvolvimento de metodologia de doseamento de cápsulas de maleato de enalapril por espectrofotometria de absorção no ultravioleta. Rev. Bras. Farm. V. 92, n. 2, p. 71-76, 2011.

LACERDA, Caroline D. Controle de Qualidade de Medicamentos Manipulados.2014. Disponível em: <http:/ / www.portaleducacao.com.br/farmacia/artigos/55623/ controle-de-qualidade-de-medicamentos-manipulados>. Acesso em:10 nov. 2015.

LAMOLHA, Marco A. et al. Avaliação da equivalência farmacêutica de furosemida em comprimidos de 40mg. Rev. Bras. Farm., v. 93, n. 1, p. 17-21, 2012.

LE HIR, A. Farmaciagalênica. Barcelona: Masson, S.A., 1995.

MARCATTO, A. P. et al. Análise de cápsulas de captopril manipuladas em farmácias. Rev. Ciênc. Farm. Básica Apl., v. 26, n.3, p. 221-225, 2005,

MALESUIK, Marcelo D. Desenvolvimento e validação de Metodologia para análise de besilato de Anlodipino em formas farmacêuticas. Dissertação (Mestrado). $130 \mathrm{f}$. Universidade de Santa Maria, 2005. Disponível em: <http:/ / cascavel.ufsm.br/tede/ tde_busca/arquivo.php?codArquivo=1553>. Acesso em: 29 abr. 2015.

RIBEIRO,Wellington; MUSCARÁ, Marcelo N. Características farmacocinéticas de antagonistas de cálcio, inibidores da ECA eantagonistas de angiotensina II em humanos. RevBrasHipertens, v. 8, n. 1, janeiro/março, 2001. Disponível em: <http:/ / departamentos.cardiol.br/dha/revista/8-1/013.pdf>. Acesso em: 12 set. 2015.

RIGOBELLO, Camila et al. Avaliação da qualidade e perfil de dissolução de comprimidos de cloridrato de propranolol. ActaScientiarum.Health Sciences,Maringá, v. 35, n. 1, p. 85-90, Jan.-June, 2013.

SARAIVA, K.I.R.O; SANTOS, Z.M.S.A.; LANDIM, F.L.P.; LIMA, H.P; SENA, V.L.O. Processo de viver do familiar cuidador na adesão do usuário hipertenso ao tratamento. Texto contexto- enferm. Florianópolis, v. 16, n. 1, p. 63-70, 2007.

SCHESHOWITSCH, Karin et al. Avaliação da Qualidade e Perfil de Dissolução de Cápsulas Manipuladas de Piroxicam. Latin American Journal of Pharmacy, v. 26, n. 5, 2007.

SILVA, Joel R. da; CHOZE, Rafael; ANDRADE, Eudes D. de; SILVA, Roberto de S. Estudo sobre partição de comprimidos de besilato de anlodipino. Ensaios e Ciência: 
Ciências Biológicas, Agrárias e da Saúde, v. 17, n. 5, 2013. Disponível em: <http:/ / www.redalyc.org/articulo.oa?id=26032926006>. Acesso em: 29 abr. 2015.

SILVERSTEIN, R. M.; BASSLER, G. C.; MORRILL, T. C. Identificação espectrométrica de compostos orgânicos. 5. ed., LTC, 2006.

SOCIEDADE BRASILEIRA DE CARDIOLOGIA (SBC). Tratamento Medicamentoso. Disponível em: <http:/ / departamentos.cardiol.br/dha/vdiretriz/08-tratamento.pdf >. Acesso em:16 maio 2015.

VILLA-JATO, J. L. Tecnologia farmacêutica. Formas Farmacêuticas. Vol I e II.Madrid. Sintesis, 1997. 